\title{
Music and neuroscience: a multidisciplinary approach to the neurobiological substrates of music related behavior George Papadelis*
}

Address: School of Music Studies, Aristotle University of Thessaloniki, Greece

* Corresponding author

from International Society on Brain and Behaviour: 2nd International Congress on Brain and Behaviour Thessaloniki, Greece. 17-20 November 2005

Published: 28 February 2006

Annals of General Psychiatry 2006, 5(SuppI I):SI doi:I0.II86/I744-859X-5-SI-SI

Two decades ago, the idea to focus on music-related aspects of behaviour as a methodological tool to study the neural correlates of human complex cognitive functions was a rather uncommon practice within neuroscience. Nowadays, a rapidly evolving field has been formed under the general name "The Neuroscience of Music" as an attempt to develop a whole new approach to the study of musical abilities, which would enrich the traditional music-theoretic ones. Besides that, and due to the fact that music-related behaviour encompasses many different functions of human cognition, it was recently proven to be a valuable window for neuroscientists onto complex brain functions. A draft map of the related research area could be formulated in terms of the simplicity or complexity of the behavioural aspect under study. That classification scheme might also be influenced by adopting a distinction between peripheral versus more centralized neural processing functions and their underlying neural structures.

To begin with the more fundamental auditory perception qualities, which also constitute the main topic of interest within the current symposium, a brief introductory discussion to the talks that follow, will be done as an attempt to reveal the common ground between all different disciplines which focus on the analysis of neural processing of stimulus's characteristics along the auditory pathway up to the cortex. This kind of rather peripheral processing is performed through a series of successive transformations and encodings, by which characteristic spectrotemporal features of acquired individual sounds or musical tones are represented by corresponding sets of elementary perceptual attributes, such as pitch, timbre, subjective timing and duration.
Recent studies on brain responses to various aspects of musical structure and syntax revealed the existence of characteristic topologies of brain activity, that correlate to specific macrotemporal aspects of music. Similarities between linguistic and musical structure processing, especially observations about the close interconnection of music and speech as an important factor for language acquisition in early life, are finally discussed through a presentation of a recently developed battery of musical tests (Overy, Ann NY Acad Sci, 2003) in combination with a computer based interface for an early diagnosis of specific learning difficulties in the domain of literacy (Overy and Papadelis, in press). 